\title{
Student Reasoning about Multivariable Covariation in Thermodynamics
}

\author{
Paul J. Emigh, Reese R. Siegel, Jonathan W. Alfson, and Elizabeth Gire \\ Department of Physics, Oregon State University, Corvallis, OR 97331
}

Understanding how quantities in multivariable relationships covary is crucial for studying thermodynamics. To study how students reason about multivariable covariation, we asked junior-level physics majors to consider an ideal gas and the corresponding contour graph of temperature $v s$. volume and pressure. We asked these students to identify how the temperature changes (a) when the volume changes and (b) when the pressure changes, without specifying how the other variable changes. We found that students used three kinds of reasoning: (1) referencing the features of the provided graph, (2) using physical knowledge about the gas, and (3) specifying a change in the third variable. Almost all students, regardless of the type of reasoning they used, answered that the temperature could only change in one way. These results suggest that instruction focusing on the different ways thermodynamic variables can change together might help students develop more sophisticated covariational reasoning skills.

2019 PERC Proceedings edited by Cao, Wolf, and Bennett; Peer-reviewed, doi.org/10.1119/perc.2019.pr.Emigh Published by the American Association of Physics Teachers under a Creative Commons Attribution 4.0 license. Further distribution must maintain attribution to the article's authors, cover page, and DOI. 


\section{INTRODUCTION}

Thermodynamics involves a large number of variables that are related to each other in rich and complicated ways. Thermal systems have a limited number of independent variables and many dependent variables. Choosing which variables are independent is not prescribed and can be done for convenience. How independent and dependence variables change - and how those changes are related to each otherare important when studying thermodynamic systems.

Covariation is the idea that the change in one variable is related to the change in another variable and is a central concept in differential calculus. Carlson et al. defines covariational reasoning as "the cognitive activities involved in coordinating two varying quantities while attending to the ways in which they change in relation to each other" [1]. A large body of mathematics education research focuses on student understanding of covariation in a variety of contexts (see, for example, Refs. [2-5]). In general, the findings of this literature suggest that covariational reasoning is complicated and difficult for many students, even in straightforward contexts.

However, prior research has predominantly focused on covariation for functions of only one variable, and very little research has been done on student understanding of multivariable covariation in either math or physics contexts. Kautz et al. [6] looked at students' understanding of the ideal gas law and found that many students tended to relate two of the variables in that law directly, ignoring possible changes in the third variable. Founds et al. [7] observed students holding the wrong variable constant when finding derivatives of multivariable functions both with and without a thermodynamics context. We have found no publications describing how students think about multivariable covariation graphically (with, for example, contour graphs).

Since both change and multivariable functions are at the heart of thermodynamics, our initial research has been guided by the following question:

Research Question: How do students reason about changes in thermodynamic variables (for a system with two independent variables)?

We explore this question by examining middle-division students' written responses to a pair of open-ended pretest questions. In this preliminary study, our aim is to characterize the space of students' ideas, with the ultimate goal of crafting curriculum to help students navigate the complicated interrelatedness of thermodynamic variables.

We begin in Sec. II by describing the student population and the task they were given. Then, Sec. III gives our methods for analyzing the data. Section IV outlines our results and Sec. V discusses these results and their implications for both further research and for instruction.
Temperature, Pressure, and Volume of Argon Gas

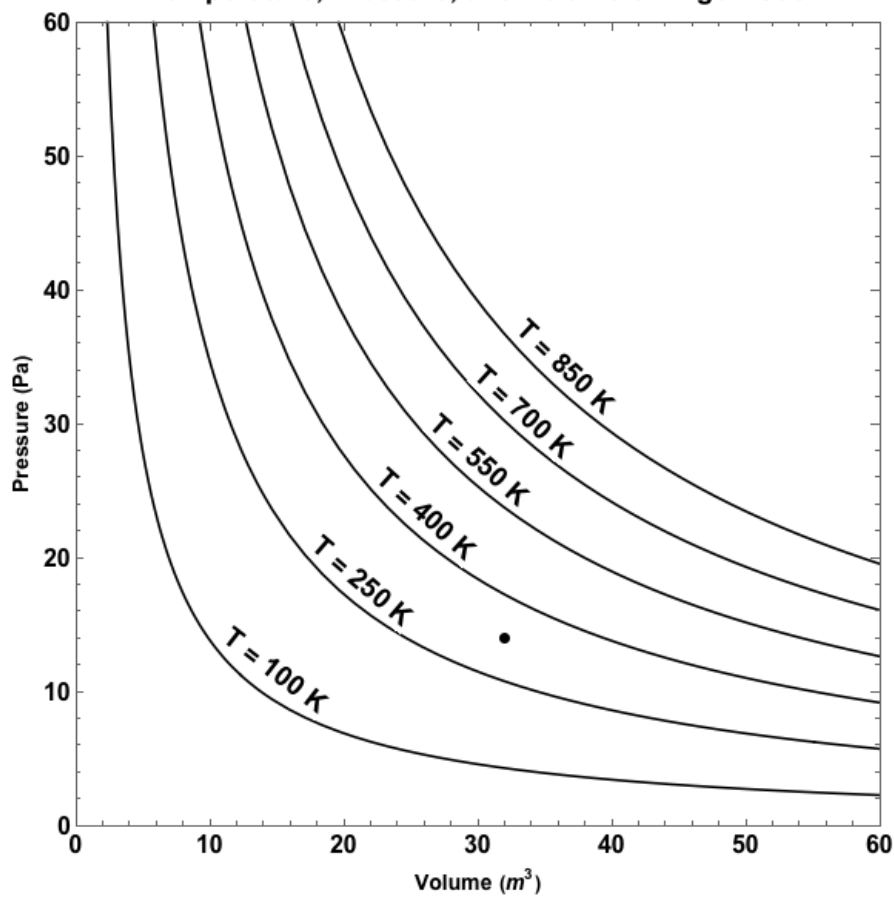

FIG. 1. A contour graph relating $T, p$, and $V$ for Argon gas, given as part of the pretest.

\section{METHODS}

This study is part of a larger project at Oregon State University (OSU) aimed at exploring and improving student reasoning about multivariable functions in physics courses $[8,9]$. This study was conducted with students enrolled in the juniorlevel thermodynamics Paradigms course at OSU [10, 11]. The students had previously completed introductory physics and multivariable calculus, but they likely had no previous instruction in thermodynamics at the college level beyond general chemistry. To get a sense of the ideas these students bring with them into this course, we developed a pretest and administered it to students $(\mathrm{N}=45)$ on the first day of class. Students received course credit for completing the pretest but not for the correctness of their answers.

On the pretest, students were shown the graph in Fig. 1 and told it "is a contour plot relating Temperature, Pressure, and Volume for Argon gas." The graph shows lines of constant temperature for a range of pressure and volume values. Students were then asked the following two questions:

Pretest Q1: At the point indicated on the plot above, suppose the Argon pressure $(p)$ increases by $15 \mathrm{~Pa}$. What can you say about the change in temperature $(T)$ ? Explain your reasoning.

Pretest Q2: At the same indicated point, as you increase the volume $(V)$ of the argon gas, how might the temperature $(T)$ change? Explain your reasoning. 
The pretest also included a third question asking students to find a rate of change for a different contour graph, which we do not include in our analysis here.

These two questions were purposefully written to be ambiguous. For example, increasing $p$ by $15 \mathrm{~Pa}$ can be done in a variety of ways (with or without holding $V$ constant), resulting in many possible changes in $T$ (including a decrease or no change). We consider this ambiguity essential to answering our research question, as we are particularly interested in learning how students deal with the multivariable nature of a thermodynamic system. Given that students often fail to attend to changes in all three variables in symbolic contexts using the ideal gas law [6], it is particularly interesting to see whether or not this remains true when students are given a graphical relationship between the variables.

\section{ANALYSIS}

We analyzed the data using a Thematic Analysis approach $[12,13]$. Multiple rounds of analysis were conducted, with each round intended to identify themes in the data. After discussion of the themes found in each round, the next round proceeded both to confirm the previously identified themes and to identify new themes that might also answer the research question. All themes came from the data itself (they were not imposed by a prior or external analysis).

The first rounds of analysis were conducted by author RRS [14]. The initial focus was to identify the individual, distinct steps in the reasoning demonstrated in the students' written responses and (separately) on the drawings students made on the provided contour graph. We then grouped these elements in several different ways in an attempt to gain insight into the underlying patterns.

Eventually, we grouped our themes according to the nature of the underlying reasoning that students used to give their general response. We found three different categories of reasoning: (1) referencing the features of the provided graph, (2) using physical knowledge about the gas, and (3) specifying a change in the third, unspecified variable ( $V$ for Q1 and $p$ for Q2). Author PJE independently reanalyzed each student's work to identify which category was most applicable, explicitly allowing for a student's work to be placed in more than one category. In this last round of analysis, we also formally identified each of the original reasoning elements as subthemes falling within one of the three larger categories, based on the underlying reasoning associated with that subtheme.

TABLE I. Student answers to the pretest questions $(\mathrm{N}=45)$.

\begin{tabular}{l||c|c|c|c|c}
\hline \hline & $T$ increases & $T$ decreases & $T$ constant & Multiple & No Response \\
\hline Q1 & 44 & 0 & 0 & 1 & 0 \\
Q2 & 38 & 4 & 0 & 2 & 1 \\
\hline
\end{tabular}

\section{RESULTS}

Virtually all students answered each question by stating that the temperature increases (see Table I). Only two students across both questions acknowledged that the temperature might change in more than one way. As discussed in Sec. III, we categorized students' reasoning into one of three categories (see Table II) according to the nature of the reasoning, based either on the graph, on physical knowledge, or on information about the unspecified variable. Below, we describe the individual reasoning elements (subthemes) associated with each of these broader categories in separate sections. We also report percentages associated with each subtheme, but we note that they should only be evaluated qualitatively due to the sample size in our study $(\mathrm{N}=45)$.

\section{A. Referencing the features of the provided graph}

The type of reasoning that we identified as the most common $(60 \%$ of the students) involved referencing the features of the provided graph. Students in this category typically provided a numerical answer for Q1, as in the following example from Q1:

\section{"T increases by $400 \mathrm{~K}$. Contour line at $(30,30)$ is $700 \mathrm{~K}$."}

As in this example, some students (24\%) explicitly identified the contour lines on the given graph that allowed them to judge the new value of the temperature. Other students $(11 \%)$ merely asserted that this information came from the graph, and some students (7\%) gave a number but did not explain how they determined this number.

\section{B. Using physical knowledge about the gas}

Many students (49\%) made use of the information given in the problem that the system is a gas (specifically, Argon gas). Most students in this category (33\% of all students) relied on the ideal gas law, though the problem did not specify that Argon can be treated as an ideal gas (it can). A common response to Q1 was:

"Using the ideal gas law, $P V=n R T$, if the pressure increases, so does the temperature."

TABLE II. Categories of student reasoning on the pretest $(\mathrm{N}=45)$. Many student responses were placed in more than one category.

\begin{tabular}{l|c}
\hline \hline Reasoning & $\%$ \\
\hline Referencing features of the provided graph & $60 \%$ \\
\hline Using physical knowledge about the gas & $49 \%$ \\
\hline Specifying a change in the third variable & $40 \%$ \\
\hline No reasoning & $4 \%$ \\
\hline
\end{tabular}




$$
\begin{aligned}
& \text { temperature will increabe to } \approx 700 K \\
& P J=k T \quad T=\frac{\rho V}{K} \quad P A \rightarrow T \uparrow
\end{aligned}
$$

FIG. 2. Student response to Q1 showing reasoning based both on the numbers given in the contour graph and on the ideal gas law.

In addition to verbal reasoning, some students who used the ideal gas law drew arrows to indicate which variables change, as shown in Fig. 2. We also note that some students who used the ideal gas law may simply have been attempting to find a symbolic solution, and that they were not considering the physical behavior of the graph explicitly. It was also the case that some students $(24 \%)$ used both the graph and the ideal gas law (as in Fig. 2).

A few students (13\%) provided alternative physical reasoning that relied on the molecular mechanics of the gas, rather than being based solely on the symbolic ideal gas law. An example is given below:

"The temperature would increase if we assume the volume is constant. This is because the molecular energy would increase as the intermolecular distance decrease."

This student appears to be reasoning about how the molecules of the Argon gas interact at a molecular level, considering both the amount of space available to the molecules and the amount of energy in the system. Students in this category used either one of these types of reasoning (space or energy) or both types of reasoning together.

\section{Specifying a change in the third variable}

We found that $40 \%$ of the students identified the third variable ( $V$ for Q1 and $p$ for $\mathrm{Q} 2$ ) and described how this third variable changes. An example of the most common type of response (in this case to Q1) is given below:

\section{"The temperature will increase, as the volume remained the same, but pressure increased."}

This student gave an answer ( $T$ increases when $p$ increases) while also asserting that the third variable, $V$, remains constant. Only one student, in response to Q2, specified that the third variable $(p)$ increases rather than stays constant. Almost all the students who gave such a response also used reasoning about either the provided graph or about the physical behavior of the gas.

One potentially important detail in these students' responses is that about half directly claimed that the third variable is constant, as in the example above. That is, the language used by the student above seems to imply that the volume must have remained the same. A contrasting example (in response to Q2) is given below:

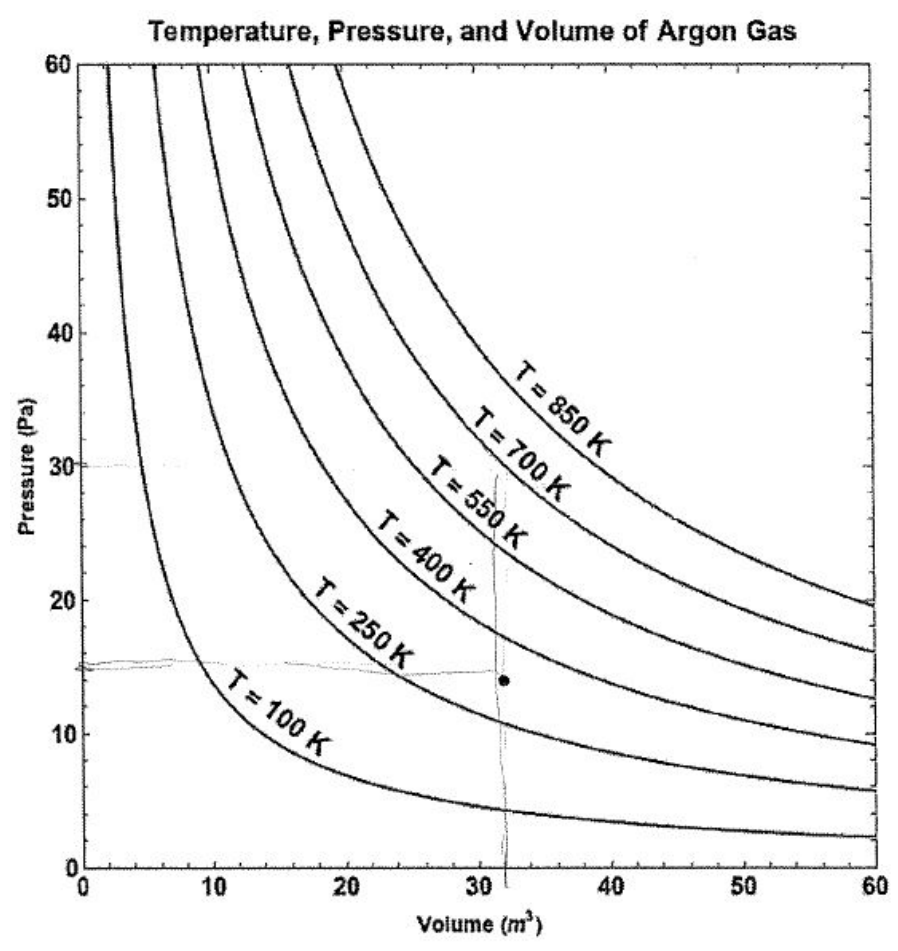

FIG. 3. A student's sketch of lines on the given contour graph.

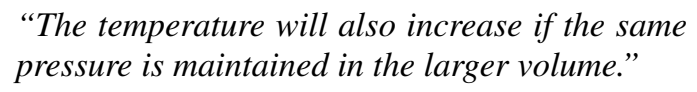

This student uses the word "if," but the responses are otherwise very similar. Each of the two response types acknowledges that the third variable is held constant, but the exact language used by the students was not explicit enough for us to determine whether or not the students truly recognized that it would be possible (in Q2, for example) to increase the volume while also making a nonzero change to the pressure. In addition to the students who discussed the third variable explicitly, we note that the answers of most other students are also consistent with holding the third variable constant.

Only one student explicitly acknowledged that there might be more than one possible change to the third, unspecified variable. Consider their response to Q2:

"If the volume increases and the pressure is held constant the temperature will increase. Like [Q1] the change in temperature depends on both pressure and volume change."

This student begins by specifying that volume changes and that pressure does not, similar to what was described above. The student then goes on to acknowledge that the change in temperature depends on both of these changes (an argument they also made in response to Q1).

In addition to using words to indicate that $V$ (in Q1) or $p$ (in Q2) might be held constant, almost half of the students (49\%) drew lines on the provided graph that leads us to believe they 
were also thinking about holding one of these variables constant. Figure 3 shows lines added to the given graph by a student. In response to Q1, this student gave a numerical answer and used the ideal gas law, but did not mention in words that $V$ should be held constant. The sketch in Fig. 3, however, suggests that the student drew a vertical line, parallel to the pressure axis, corresponding to the appropriate change in pressure, to help find a numerical value for the change in temperature (they answered that the "temperature increases from about $300 \mathrm{~K}$ to $650 \mathrm{~K}$ "). This student's response to Q2 was "Temperature increases if pressure is kept constant," further suggesting that the sketch may indicate that $V$ is constant for Q1. For many such responses, though, we cannot be sure whether or not any given sketch indicates the student was thinking about holding a variable constant.

\section{DISCUSSION AND IMPLICATIONS}

In providing a contour graph of temperature vs. pressure and volume, we expected almost all students to base their answer on the graph. We were therefore surprised so many students $(49 \%)$ reasoned based on some physical knowledge of the behavior of the gas. We view this result primarily as evidence that students are able to draw on a diverse set of resources when answering questions about physical systems, which is already something we aim to cultivate in instruction. However, since $38 \%$ of the students did not reference the provided graph at all, it is possible that students are too unfamiliar or uncomfortable with contour graphs to rely on them for this task.

Overall, our results show that many students acknowledged the multivariable nature of the context in one of two ways. First, a combined $42 \%$ of the students mention the third, unspecified variable in some way-almost always to assert or suppose that this variable is held constant. Second, $49 \%$ of the students drew horizontal and/or vertical lines on the graph. However, students who drew such a line without any supporting reasoning may or may not have been thinking explicitly about holding a variable constant (a total of $18 \%$ of the students did both). Whether they wrote words or drew lines, these students reasoned about the change in temperature when one of the independent variables changes and the other independent variable is constant. This kind of reasoning strikes us as a possible "default" way to think about a function of two variables. Graphically, we believe that thinking of a change in one variable as corresponding to moving strictly in the direction of that variable's axis, perpendicular to the other axis, is natural. It seems similarly natural to us that students might typically assume for a symbolic equation that all variables are held constant unless specified otherwise, a pattern of behavior that we have observed in other settings [7].

In thermodynamics, however, the variables are not spatial, and the variables cannot really be thought of as naturally orthogonal to each other. Changes of state in which more than one independent variable changes are not only possible, they are universal, and it is essential that students be able to reason about such changes. Our data, however, suggests that students are "stuck" on the idea that one axis variable must be held constant when reasoning about changes in variables in thermodynamics. Nearly all students did not reason about any other possible changes in response to these prompts.

Promoting reasoning in which both axis variables change strikes us as a particularly rich idea for improving instruction. We suggest leveraging the reasoning that students appear to do spontaneously to help broaden the set of changes students are willing to consider. The prevalence of students noting that the third variable is constant seems like a promising step toward building more sophisticated reasoning about multivariable covariation. We suggest providing students with a context where they can give reasoning like this, followed by an opportunity to consider either what would happen if the third variable is not constant or how the state could be changed to produce a different change in the dependent variable. We also suspect that following this line of questioning by asking students about a contour graph in which four variables are represented simultaneously (e.g., adding lines of constant entropy to Fig. 1) would help solidify an understanding of multivariable relationships in thermodynamics by making the existence of additional paths (holding different variables constant) more apparent. Furthermore, since some students appear comfortable reasoning with graphs and some with equations and physical behavior, exposing groups of students to context-rich problems is likely to give students exposure to different ideas and to broaden the set of representations with which any particular students is comfortable. We are currently developing and investigating the effectiveness of curriculum that uses multiple representations, including contour graphs and plastic surfaces, to help students consider changes along directions other than those parallel and perpendicular to the axes [8].

We note that there are some obvious limitations to this study. The data consists of students' written work, and thus we were not able to ask follow-up questions to probe students' reasoning in more detail. The questions were also given at the beginning of a thermodynamics course, so our results do not indicate how the sophistication of the students' reasoning might be different after junior-level thermodynamics. We are currently conducting a parallel study of students' in-class responses to prompts similar to those on the pretest aimed at exploring students' ideas_and how they might shift as a result of instruction-in greater detail [8].

\section{ACKNOWLEDGMENTS}

We thank the OSUPER group, especially Abigail Kimbrough and Michael Trumbull, along with Aaron and Robyn Wangberg, and the students who participated in the study. This work was supported by NSF grants DUE-1323800 and DUE-1612480. 
[1] M. P. Carlson, S. Jacobs, E. Coe, S. Larsen, and E. Hsu, Applying covariational reasoning while modeling dynamic events: A framework and a study, J. Res. Math. Educ. 33, 352 (2002).

[2] P. W. Thompson, The development of the concept of speed and its relationship to concepts of rate, in The development of multiplicative reasoning in the learning of mathematics (SUNY Press, 1994).

[3] J. Confrey and E. Smith, Splitting, covariation, and their role in the development of exponential functions, Journal for Research in Mathematics Education 26 (1995).

[4] K. C. Moore, Quantitative reasoning and the sine function: The case of Zac, Journal for Research in Mathematics Education 45 (2014).

[5] W. Hung and D. H. Jonassen, Conceptual understanding of causal reasoning in physics, International Journal of Science Education 28, 1601 (2006).

[6] C. H. Kautz, P. R. Heron, M. E. Loverude, and L. C. McDermott, Student understanding of the ideal gas law, part i: A macroscopic perspective, American Journal of Physics 73, 1055 (2005)

[7] I. W. Founds, P. J. Emigh, and C. A. Manogue, Student responses to chain rule problems in thermodynamics, in Physics Education Research Conference 2017, PER Confer- ence (Cincinnati, OH, 2017)

[8] J. W. Alfson, P. J. Emigh, R. R. Siegel, R. Wangberg, A. Wangberg, and E. Gire, Explicitly prompting covariational reasoning in a thermodynamics context, in Proceedings of the Physics Education Research Conference 2019 (submitted).

[9] A. Kimbrough, P. J. Emigh, J. W. Alfson, and E. Gire, Teaching gravitational potential energy: Student interaction with surface manipulatives, in Proceedings of the Physics Education Research Conference 2019 (submitted).

[10] C. A. Manogue and T. Dray, Functions (Bridge Project website), http://math.oregonstate.edu/bridge/ideas/functions (2002).

[11] C. A. Manogue and K. S. Krane, The Oregon State University Paradigms Project: Re-envisioning the upper level, Phys. Today 56, 53 (2003).

[12] J. Aronson, A pragmatic view of thematic analysis, The qualitative report 2, 1 (1995).

[13] V. Braunand V. Clarke, Using thematic analysis in psychology, Qualitative research in psychology 3, 77 (2006).

[14] R. R. Siegel, Understanding change: Surface manipulativeactivities as a tool for addressing student difficulties in thermodynamics (2019). 